\title{
Anterior segment ischaemia following segmental scleral buckling
}

\author{
Jeffrey Kwartz, Stephen Charles, Paul McCormack, Alan Jackson, Michael Lavin
}

Manchester Royal Eye Hospital, Oxford Road, J Kwartz

$S$ Charles

P McCormack

M Lavin

Department of

Neuroradiology,

Manchester Royal Manchester M13 9WL

A Jackson

Correspondence to: Mr Jeffrey Kwartz. 24 November 1993 Manchester M13 9WH

Infirmary, Oxford Road,

Accepted for publication

Anterior segment ischaemia is a well recognised complication following retinal detachment surgery. This case report describes postoperative anterior segment ischaemia in a patient with known atheromatous disease of the intracranial vessels.

\section{Case report}

A 55-year-old white man presented with an 8 month history of poor vision in the left eye. $\mathrm{He}$ had a history of mild asthma and 2 years previously had suffered a subarachnoid haemorrhage caused by a left middle cerebral artery aneurysm which was subsequently clipped. There was no visual disturbance associated with the subarachnoid haemorrhage.

On examination the right eye was normal but left visual acuity was reduced to counting fingers. Funduscopy revealed a left subtotal retinal detachment, involving the macula, associated with two small retinal breaks in the inferotemporal periphery.

Retinal reattachment surgery was performed under general anaesthesia. A $360^{\circ}$ conjunctival limbal peritomy was performed, the retinal holes were localised, and transcleral cryopexy to the retinal breaks was performed. Subretinal fluid was drained below the lateral rectus muscle and

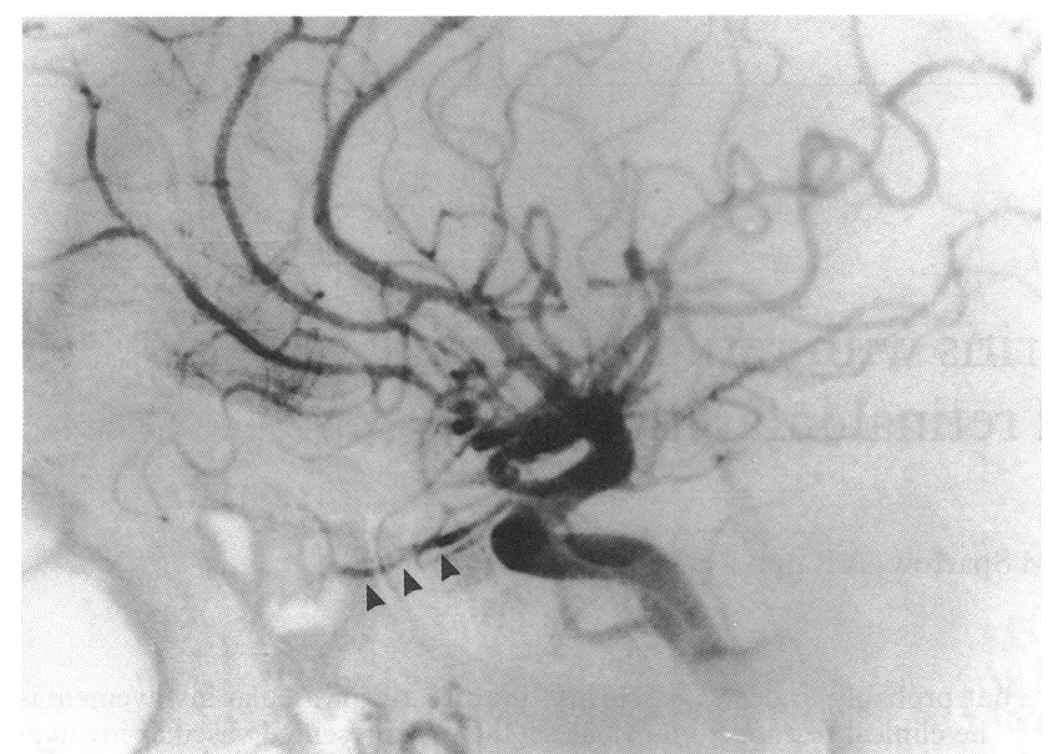

Figure 1 Right common carotid angiogram demonstrating a normal orbital portion of the ophthalmic artery (arrowheads). ocular volume replacement was attained with an injection of sterile air. A circumferential 277 silicone episcleral explant was secured to the whole of the inferotemporal quadrant. Patency of the central retinal artery was confirmed, the peritomy was closed, and a subconjunctival injection of cephradine given with atropine drops instilled into the eye. The patient was positioned with right cheek to pillow.

On the first day postoperatively, the left cornea was very oedematous with marked folding of Descemet's membrane. The anterior chamber was deep with flare present and a dilated pupil (post atropine). The intraocular pressure was $2 \mathrm{~mm} \mathrm{Hg}$. There was a good red reflex and the retina appeared to be flat. A diagnosis of anterior segment ischaemia was made.

The patient was treated with hourly topical dexamethasone drops. Ultrasonic corneal pachymetry was performed, which showed a normal right corneal thickness $(556 \mu \mathrm{m})$ and increased left corneal thickness $(1032 \mu \mathrm{m})$. The left corneal oedema gradually subsided and by 4 months the cornea was clinically normal (ultrasonic pachymetry $587 \mu \mathrm{m}$ ). There was mild iris atrophy, normal intraocular pressure, and the retina was attached. Left visual acuity was $2 / 60$ because of post detachment atrophy.

The cerebral angiograms taken just before neurosurgical intervention 2 years previously have subsequently been reviewed. These show a normal patency of the right ophthalmic artery (Fig 1). On the left, ipsilateral to the anterior segment ischaemia, there is occlusion of the ophthalmic artery (Fig 2), with a collateral blood supply to the central retinal artery from branches of the maxillary division of the external carotid artery. It has subsequently been revealed that following neurosurgery the patient suffered a mild but transient dysphasia and left ptosis.

\section{Comment}

Anterior segment ischaemia is a well described but uncommon complication following retinal detachment surgery. Predisposing factors include surgical manoeuvres such as an encircling scleral buckle, disinsertion of ocular muscles, and diathermy or cryotherapy to the long posterior ciliary vessels or an underlying systemic vascular or haematological condition such as haemoglobin S-C disease which may compromise ocular blood flow. ${ }^{1}$

The pathogenesis of anterior segment ischaemia following retinal detachment surgery 


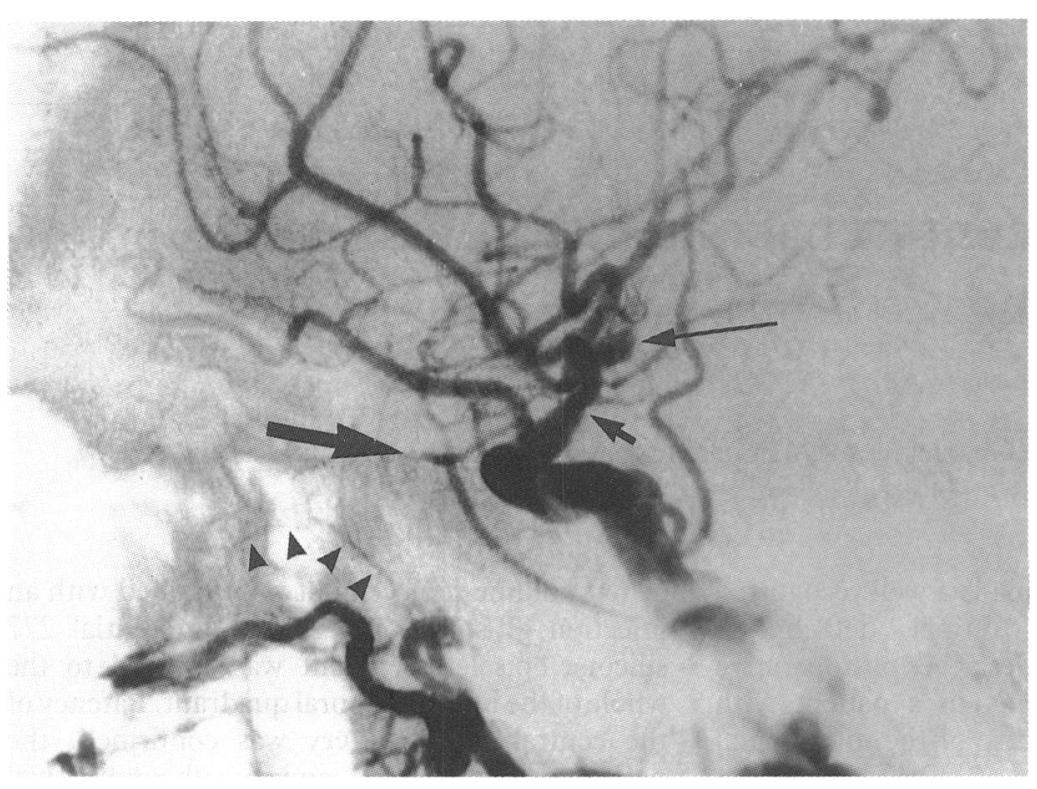

Figure 2 Left common carotid angiogram demonstrating left middle cerebral artery aneurysm (long arrow). There is a quite severe atheromatous stricture of the post cavernous internal carotid (short arrow). The ophthalmic artery is occluded immediately distal to the optic canal (large arrow) and the central retinal artery is fed by branches of the maxillary division of the external carotid artery (arrowheads).

has been attributed to several factors, including interruption of the anterior ciliary arteries, damage to the long posterior ciliary arteries, and compression of vortex veins reducing uveal blood flow. ${ }^{2}$ In our case there was no encircling element placed around the eye and no interference with the anterior or posterior ciliary vessels. Anterior segment ischaemia has only rarely been described following use of a segmental buckle. ${ }^{3}$

Chronic anterior segment ischaemia as part of the panocular ischaemia seen with carotid occlusive disease has been described. ${ }^{4}$ In the case we report it is suggested that the atheromatous disease of the internal carotid artery demonstrated previously, with presumably an associated reduction in ophthalmic artery flow, was of significance in the precipitation of acute anterior segment ischaemia following routine nonencircling retinal detachment surgery. The sub- arachnoid haemorrhage and need for aneurysm clipping is not thought to be of significance in the precipitation of anterior segment ischaemia but has provided angiographic evidence of significant co-existing atheromatous disease.

Collateral circulations in the anterior segment have been described, including superficial marginal plexus at the limbus, episcleral limbal plexus, the intramuscular circulation in the ciliary body, and the major arteriolar circle in the iris root. Experimental evidence in animals has been presented to show that a $360^{\circ}$ peritomy has a demonstrable effect in the production of anterior segment ischaemia following strabismus surgery. ${ }^{5}$ Our patient may have had a precarious ocular blood supply before detachment surgery further compromised by the $360^{\circ}$ conjunctival peritomy sufficient to interrupt the blood supply to his anterior segment.

It has been shown that the blood flow rates in the major temporal retinal arteries following uncomplicated scleral buckling is on average $50 \%$ lower than in the unoperated contralateral eye. ${ }^{6}$ Removal of the scleral buckling element has been shown to increase arterial blood flow rates. In the presence of suspected predisposing factors such as carotid occlusive disease appropriate measures may be taken to avoid anterior segment ischaemia. These include a smaller and more posterior conjunctival incision and adopting an appropriate vitreoretinal approach that avoids extensive buckling and encircling procedures.

l Ryan SJ, Goldberg MF. Anterior segment ischemia following scleral buckling in sickle cell hemoglobinopathy. Am $\mathcal{F}$ Ophthalmol 1971; 72: 35-50.

2 Hayreh SS, Baines JAB. Occlusion of the vortex veins. Br f Ophthalmol 1973; 57: 217-38.

3 Robertson DM. Anterior segment ischemia after segmental episcleral buckling and cryopexy. Am f Ophthalmol 1975; 79. $871-4$.

4 Dugan JD, Green WR. Ophthalmic manifestations of carotid occlusive disease. Eye 1991; 5: 226-38.

5 Fishman PH, Repta MX, Green WR, D'Anna SA, Guyton DL. A primate model of anterior segment ischemia after A primate model of anterior segment ischemia

6 Ogasawara H, Feke GT, Yoshida A, Milbocker MT, Weiter JJ McMeel JW. Retinal blood flow alterations associated with scleral buckling and encircling procedures. Br $\mathcal{F}$ Ophthalmol 1992; 76: 275-9.
Department of Ophthalmology, University of Leicester, Leicester Royal Infirmary

J M Sparrow

A R Rosenthal

Correspondence to: Mr N A Frost, Department of Ophthalmology, Leicester Royal Infirmary, Leicester LE1 5WW.

Accepted for publication 13 December 1993

\title{
Posterior scleritis with retinal vasculitis and choroidal and retinal infarction
}

\author{
N Andrew Frost, John M Sparrow, A Ralph Rosenthal
}

Posterior scleritis is a rare but probably underdiagnosed disease process. The clinical features are variable and the lesions may cause diagnostic confusion. Visual impairment is common but visual loss due directly to vascular involvement is unusual. In the case presented vascular involvement was exceptionally severe and determined the long term visual outcome. 